\title{
Nanoscale surface chemistry in self- and directed-assembly of organic molecules on solid surfaces and synthesis of nanostructured organic architectures ${ }^{\star}$
}

\author{
Feng Tao \\ Department of Chemistry, Princeton University, Princeton, NJ 08544, USA
}

\begin{abstract}
This article briefly reviews the interplay of weak noncovalent interactions involved in the formation of self-assembled monolayers of organic molecules and the strong chemical binding in directed-assembly of organic molecules on solid surfaces. For a self-assembled monolayer, each molecule involves at least three categories of weak interactions, including molecule-substrate interactions, molecule-molecule interactions in a lamella, and molecule-molecule interactions between two adjacent lamellae. Basically, molecule-substrate interactions play a major role in determining molecular configuration. Molecule-molecule interactions, particularly the interactions of molecular ending functional groups between two adjacent lamellae, such as hydrogen bonds, play a dominant role in determining the molecular packing pattern in a monolayer. These weak interactions may induce or influence molecular chirality. This understanding at the atomic scale allows us to design 2D nanostructured organic materials via precisely manipulating these weak noncovalent interactions. Compared to the self-assembled monolayer formed via weak noncovalent interactions, the structure of directed-assembled monolayer/multilayers formed through strong chemical bonds is significantly dependent on the geometric arrangement and reactivity of active sites on the solid surface. In contrast to the significant role of weak intermolecular interactions in determining molecular packing in a self-assembled monolayer, strong chemical binding between molecules and reactive sites of a substrate plays a major role in determining the molecular packing pattern in a directed-assembly monolayer. Controllable chemical attachment between organic functional groups and reactive sites of the solid surface is crucial for the formation of a highly oriented organic monolayer and the following multilayer.
\end{abstract}

Keywords: scanning tunneling microscopy; STM; self-assembly; chirality; surface chemistry; semiconductor surface; nanoscale; silicon surface; directed assembly; odd-even effect.

\section{INTRODUCTION}

Methods for creating advanced materials such as 2D and 3D nanostructured materials and devices using chemical approaches typically include both molecular self-assembly through weak noncovalent interactions and directed-assembly of molecules via strong chemical bonds with solid surfaces. These assembly techniques of organic molecules on solid surfaces form an important basis for a wide spectrum

*Pure Appl. Chem. 80, 1-57 (2008). A collection of invited, peer-reviewed articles by the winners of the 2007 IUPAC Prize for Young Chemists. 
of promising applications, including molecular electronic devices, biomolecular recognition chips, tribology, corrosion inhibition, and 3D nanopatterning. The application of the self- and directed-assembly to the syntheses of nanoscale materials and devices is determined by a thorough understanding of various surface chemistries at the nanoscale involved in these assembly processes. These assemblies originate from interfacial noncovalent interactions and/or surface chemical reactions at molecular or atomic scale. The surface science involved in these techniques and applications is termed nanoscale surface science. Although a tremendous number of studies have been carried out in the syntheses and applications of nanostructured organic/inorganic $1 \mathrm{D} / 2 \mathrm{D} / 3 \mathrm{D}$ materials and devices via these assembly techniques, the nanoscale surface chemistry and the intrinsic connection between the surface chemistry and materials syntheses and structures have been less extensively addressed.

Here, the nanoscale surface chemistry occurring in the self- and directed-assembly of various organic molecules on solid surfaces was experimentally explored; nanostructured organic architectures were synthesized on solid surfaces; new synthetic methodologies which produce nanostructured organic architectures were developed [1]. The experimental techniques used here to study the nanoscale surface chemistry of these assembled systems are a homemade scanning tunneling microscope (STM) and a homebuilt high-resolution electron energy loss spectrometer (HREELS). This review is organized with four sections including (1) molecule-substrate interactions-induced variation of molecular configuration; (2) molecule-molecule interactions-determined packing structure of organic molecules in self-assembled monolayers; (3) syntheses of nanostructured organic architectures on solid surfaces by molecular self-assembly via precisely controlling weak noncovalent interactions; and (4) surface chemistry and mechanism of directed-assembly of organic molecules via strong chemical bonds and growth of organic architectures. It is noted that this review is mainly based on the author's dissertation research and therefore many topics related to this issue will not be included, though there is a large amount of excellent work of other groups in the literature related to these topics.

\section{MOLECULE-SUBSTRATE INTERACTIONS-INDUCED VARIATION OF MOLECULAR CONFIGURATION}

Here, the self-assembly of several categories of organic molecules on solid surfaces was used as a model system for mechanistically understanding possible modification of molecular configuration induced by molecule-substrate interactions at the atomic level. As schematically shown in Fig. 1, for each molecule of a self-assembled monolayer formed on a solid surface its adsorption includes at least three categories of noncovalent forces. They are the interactions between two adjacent molecules in a lamella, the interactions between adjacent molecules of two neighboring lamellae, and the interactions between molecules and the solid surface. These interactions determine molecular configuration and packing pattern in the self-assembled monolayer.

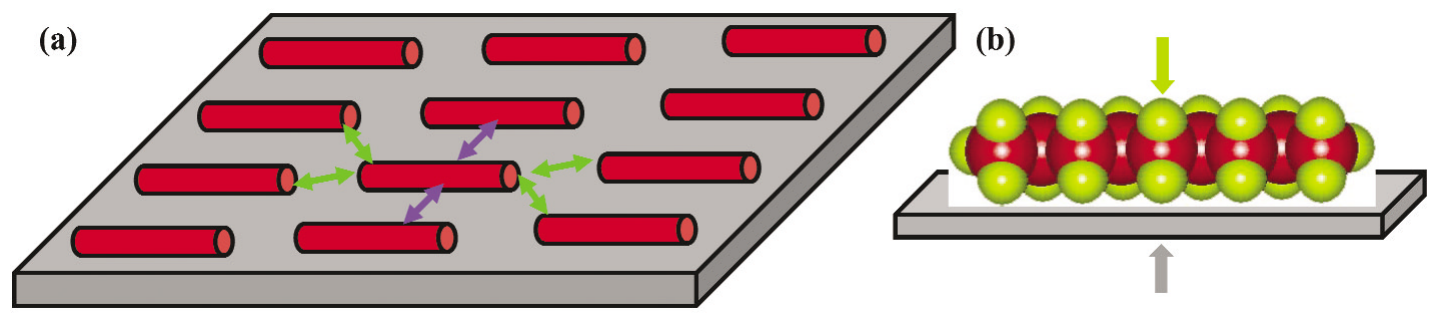

Fig. 1 (a) Scheme of intermolecular interactions in a lamella and intermolecular interactions between two adjacent lamellae. (b) Side view of a self-assembled monolayer showing molecule-substrate interactions. Each red bar shows an organic molecule. 
Recent studies revealed that molecule-substrate interactions play a major role in modifying molecular configuration upon self-assembly. For example, recent studies [2,3] showed that lattice match between the $n$-alkyl chain of organic molecules such as ester and di-alcohol and highly oriented pyrolitic graphite (HOPG) results in a modification of molecular configuration, further inducing new chirality for achiral molecules. In the solid or liquid phase of an ester, the two alkyl chains of one ester molecule are offset from each other (Fig. 2a). However, the originally bent ester molecule is distorted into a linear configuration upon self-assembly on graphite (Fig. 2b). The linear configuration was clearly identified in STM images of the self-assembled ester monolayers with atomic resolution (Fig. 3). The observed self-assembled lamellae of the ester molecules are very similar to those patterns typically observed for long-chain $n$-alkanes [4,5]. Each individual molecule has a linear shape as marked with the red dashed line in Fig. 3. Six ball-stick models of the ester molecules are superimposed on the image in Fig. 3b. Five of them are distorted into a linear shape. The sixth model has the originally bent structure of the ester molecule, in which one alkyl chain bends from the other at the ester linkage. It is clear that the first five linear models match quite well with their underlying molecular images at an atomic-resolution level. However, the model with the original bent structure does not match with its underlying image, as one of its alkyl chains obviously offsets from the underlying image. By comparing the originally bent molecular structure to the observed molecular image, it is clearly seen that the ester molecules distort into a linear shape when they self-assemble on graphite. This distortion was also observed in the self-assembled monolayers of other esters with different chain length such as lauric acid palmityl ester and lauric acid behenyl ester [2]. The excellent match of the linear molecular model with the observed molecular image clearly shows that these esters distort into a linear shape upon self-assembly on graphite.

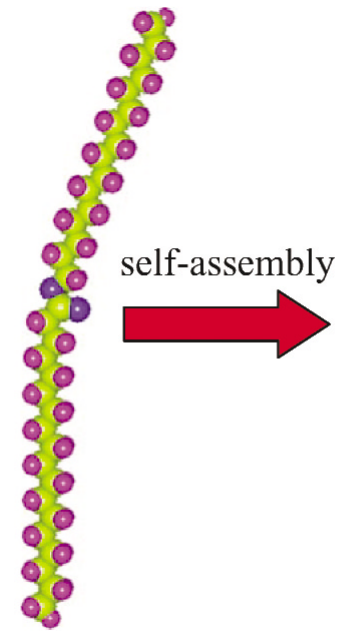

(a)

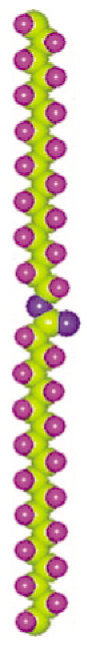

(b)

Fig. 2 Scheme showing the distortion of an ester molecule from the originally bent configuration (a) into a linear configuration (b) upon self-assembly. 


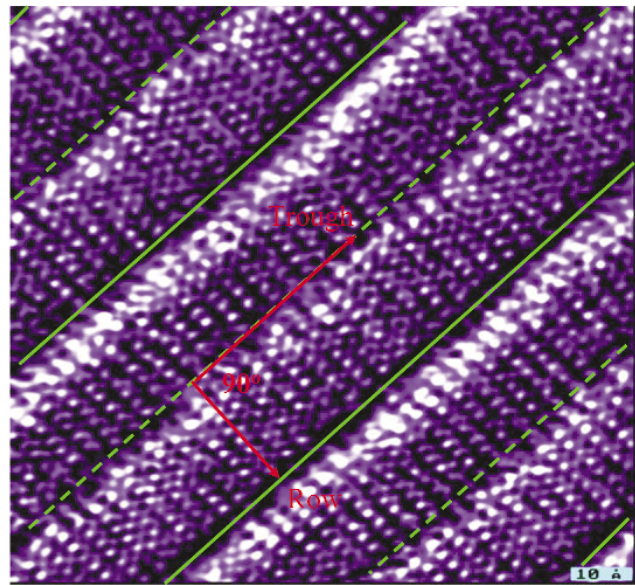

(a)

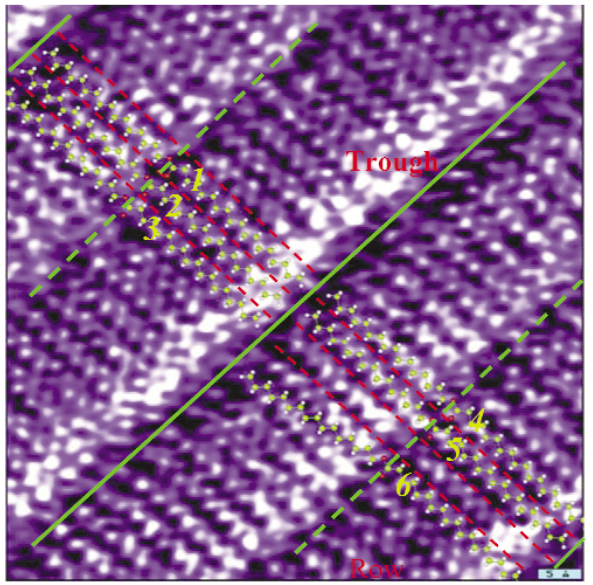

(b)

Fig. 3 STM image of the self-assembled stearic acid palmityl ester molecules on HOPG with atomic resolution. In image $b$, the superimposed molecular models 1-5 with a linear shape match with the molecular images under them. Molecular model 6 with an originally bent conformation clearly offsets from the image under it.

Considering the two possible directions for the alkyl chains bonded at the carboxylic group, two possible conformations ( $a p$ and $s p$ ) for the carboxylic ester can be expected [6]. The $\mathrm{n} \rightarrow \pi^{*}$ delocalization makes the ester group planar for both $a p$ and $s p$ conformations [6]. Figure 4 presents the optimized $a p$ and $s p$ conformations of stearic acid palmityl ester obtained with Hyperchem 7.0. Density functional theory (DFT) calculations indicated that the structure upon linear distortion has energy higher than the ap-conformation by $10.5 \mathrm{~kJ} \mathrm{~mol}^{-1}$ and than the $s p$-conformation by $7.2 \mathrm{~kJ} \mathrm{~mol}^{-1}$ [7].

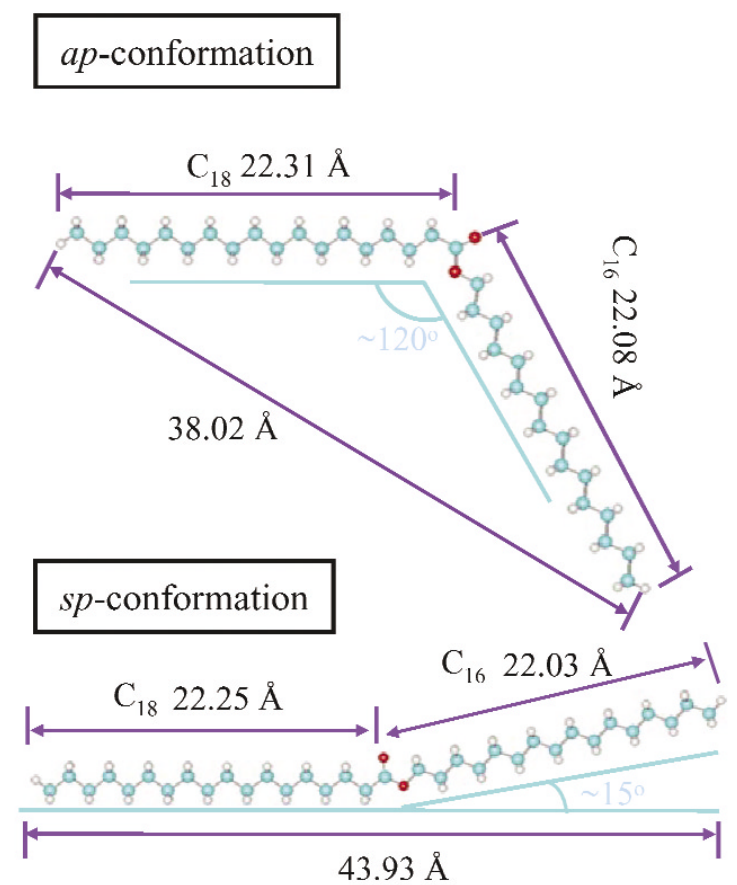

Fig. $4 a p$ and $s p$ conformations of stearic acid palmityl ester molecules. 
Obviously, the distortion induces some strain and produces a relatively unstable linear configuration. However, all the three esters in the self-assembled monolayers do distort into the linear shape on graphite. The energy cost to distort the molecular chain is compensated by the increased chain-substrate interactions resulting from the maximized molecule-substrate interactions upon distorting into a linear shape.

Another interesting example is molecular distortion from a linear shape into a bent configuration. Figure 5a shows a homogeneous herringbone structure of $d i$-ol $\mathrm{HOCH}_{2}\left(\mathrm{CH}_{2}\right)_{14} \mathrm{CH}_{2} \mathrm{OH}$. Each lamella has a chain-to-trough angle of $\sim 60^{\circ}$ (or $\sim 120^{\circ}$ ) along the clockwise direction. Correspondingly, each adjacent lamella has a clockwise chain-to-trough angle of $120^{\circ}\left(\right.$ or $\left.60^{\circ}\right)$. The two supplementary angles result from two opposite packing faces of this di-ol molecule on HOPG. Figure 5b presents the hydrogen-bonding networks at each trough between the two adjacent lamellae. Notably, lamellae 1, 3, 5, and $\mathbf{7}$ are brighter than lamellae $\mathbf{2 , 4 , 6}$, and $\mathbf{8}$. Further close examination indicates that on average the two ends of lamellae $\mathbf{2}, \mathbf{4}, \mathbf{6}$, and $\mathbf{8}$ have higher contrast than the center parts of these lamellae. Line-profile analyses in Fig. 5c show lamellae 1, 3, 5, and $\mathbf{7}$ are obviously higher than lamellae 2, 4, 6, and $\mathbf{8}$. Similar line-profile analyses for the sections from the right end of lamella 2 (or 4 ) to the left end of lamella 4 (or 6) were carried out. These analyses suggest that the alternate variation of contrast might result from a distortion of the alkyl chain upon adsorption on the surface. This distortion is possibly similar to that seen for alkyl chains of all-trans unbranched alkanes and 1-octadecanol upon self-assembly on HOPG [4,8-10].

The main driving force for the formation of stable self-assembled monolayers of all-trans unbranched alkanes and their derivatives on HOPG is the good match between the graphite lattice unit $(2.46 \AA)$ and the $\mathrm{H}-\mathrm{H}$ distance of two adjacent metylene groups $(2.52 \AA)$ in the hydrogen chain. However, there is still a $2.5 \%$ mismatch [10]. To keep good registry with the substrate lattice and to maximize van der Waals interaction, a compression of $2.5 \%$ of the alkane chain length is advantageous. This compression distorts the molecular chain in the plane perpendicular to the graphite surface. The distortion of the molecular chain does cost energy, while the reduction of the chain length lowers the overall energy due to a better adsorbate-substrate interaction. How much the chain is compressed depends on the balance of these two opposite energetic effects. Theoretical calculation could be a good approach to address this issue.

Based on understanding of height variation along the backbone of long-chain alkane molecules [11], the formation of alternate bright and dim lamellae of Fig. 5a likely results from the slight distortion of the molecular chain of $\mathrm{HOCH}_{2}\left(\mathrm{CH}_{2}\right)_{14} \mathrm{CH}_{2} \mathrm{OH}$ due to its compression and preservation of the planar configuration of the $\mathrm{O}-\mathrm{H} \cdots \mathrm{O}-\mathrm{H}$ hydrogen bonds. For example, when the center part of lamella 2 bends down, its $\mathrm{OH}$ groups tilt up (Fig. 5d). To preserve the planar configuration of $\mathrm{O}-\mathrm{H} \cdots \mathrm{OH}$ hydrogen bonds formed with its adjacent molecules in lamellae $\mathbf{3}$, the HO groups of lamella $\mathbf{3}$ have to tilt down, and the center part bends up. For the same reason, the other ends of these molecules (lamella 3 ) tilt down. Therefore, to keep the planar configuration of the hydrogen bonds molecules in lamella 3 bridge over those in lamellae 2 and 4 . Figure $5 \mathrm{~d}$ is a model of three molecules in this bridge-like configuration, in which molecules of lamella $\mathbf{3}$ bridge over the left- and right-end of molecules from lamellae $\mathbf{4}$ and $\mathbf{2}$, respectively. The formed structure is a compromise between maximizing the adsorbate-substrate interactions and preserving the planar configuration of hydrogen bonds in a unit cell. The alternate asymmetric distortion is confirmed by line-profile analyses as shown in Fig. 5c. Clearly, the adsorbate conformation is different from that of the molecule in the liquid or solid phases. The unit cell of this image shown in Fig. 5d is a pair of hydrogen-bonded molecules from two adjacent lamellae. Since they are asymmetrically distorted, the symmetry is broken. Thus, this pair is chiral. Furthermore, the two adjacent lamellae composed of ordered chiral pairs are chiral. The domain made of ordered arranged chiral lamellae is a chiral domain. This is similar to the induced chiral domain of 1-octadecanol driven by asymmetric distortion of two adjacent lamellae [10]. 

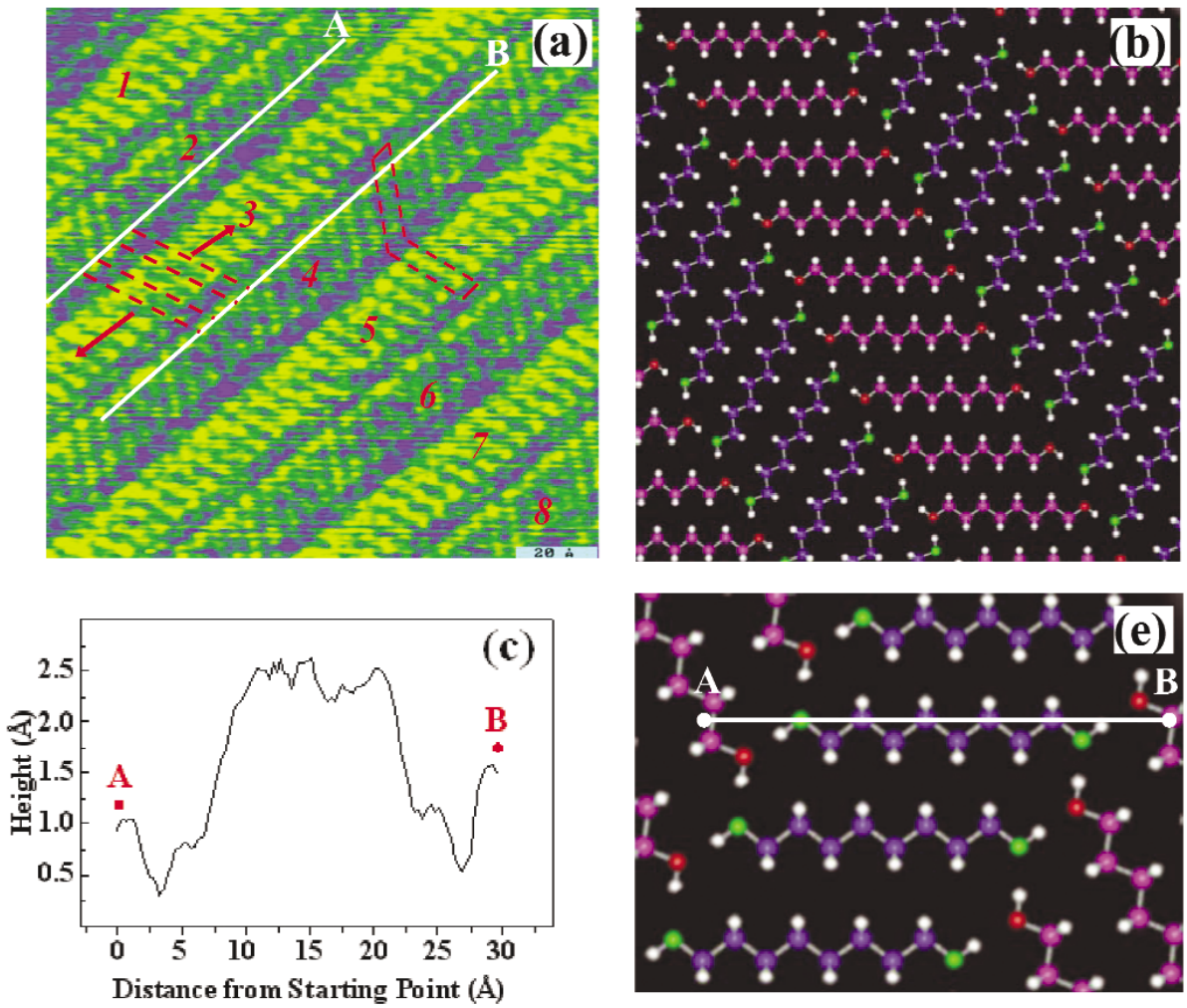

(d)

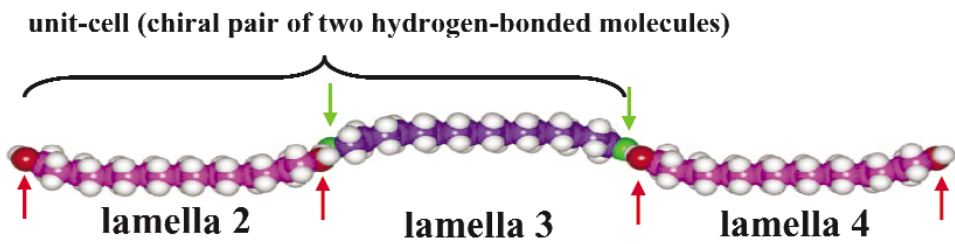

Fig. 5 (a) One image of $\mathrm{HOCH}_{2}\left(\mathrm{CH}_{2}\right){ }_{14} \mathrm{CH}_{2} \mathrm{OH}$ dissolved in octanoic acid. $140 \times 140 \AA$. (b) Schematic presentation for the herringbone hydrogen-bonding network corresponding to this image. (c) The average height profiles of 20 sections in terms of 20 molecules as marked with red dashed lines along the chain direction of $\mathrm{HOCH}_{2}\left(\mathrm{CH}_{2}\right)_{14} \mathrm{CH}_{2} \mathrm{OH}$ in lamellae 3 of Fig. 5a. The starting point A and ending point $\mathrm{B}$ of line-profile analyses are on the left and right white lines of Fig. 5a. The line-profile section from A to B is also schematically marked in Fig. 5e. (d) Schematic presentation for the bending-up and -down distortion of molecules in adjacent lamellae. The arrows show the bending directions. Notably, the herringbone structure of this di-ol cannot be seen in this scheme though it was clearly observed in the STM image (Fig. 5a). This scheme exaggerated the bending for a clear view. (e) Scheme for the starting and ending points of line-profile analyses of Fig. $5 \mathrm{c} . \mathrm{HOCH}_{2}\left(\mathrm{CH}_{2}\right)_{6} \mathrm{CH}_{2} \mathrm{OH}$ represents $\mathrm{HOCH}_{2}\left(\mathrm{CH}_{2}\right)_{14} \mathrm{CH}_{2} \mathrm{OH}$ for a clearer presentation.

\section{MOLECULE-MOLECULE INTERACTIONS-DETERMINED PACKING STRUCTURE OF ORGANIC MOLECULES IN SELF-ASSEMBLED MONOLAYERS}

The intermolecular interactions, particularly the interactions of molecular functional groups, play a dominant role in determining the molecular packing pattern in self-assembled systems. For example, the shape and size of the anhydride group of arachidic anhydride make this molecule adopt an unusual interdigited packing pattern to maximize molecular coverage on graphite [12]. In this packing, only when all molecules in a domain use the same face to pack on the surface, the molecular coverage on 
this surface can be maximized (Fig. 6). Thus, each domain is an enantiomer. The self-assembly of this molecule forms two 2D chiral structures with opposite chirality (Figs. 6 and 7).
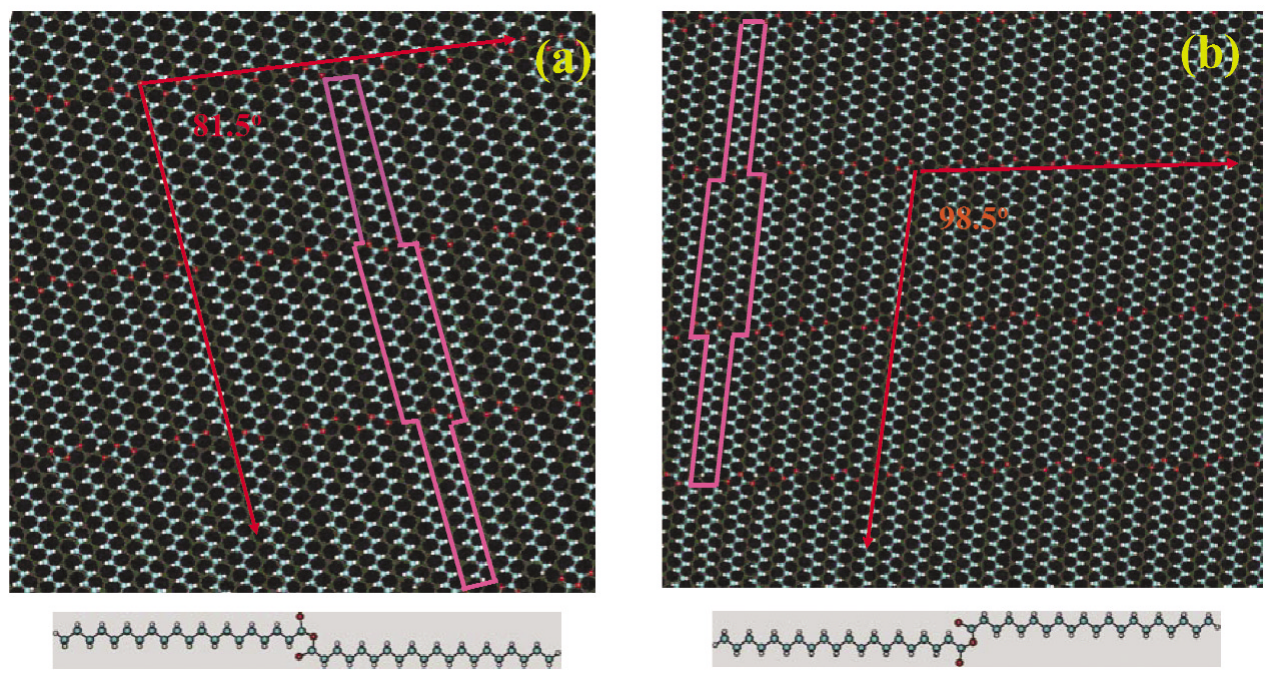

Fig. 6 Molecular arrangements with the same face to maximize molecular coverage on surface. In structures $a$ and $b$, molecules use opposite faces to pack.
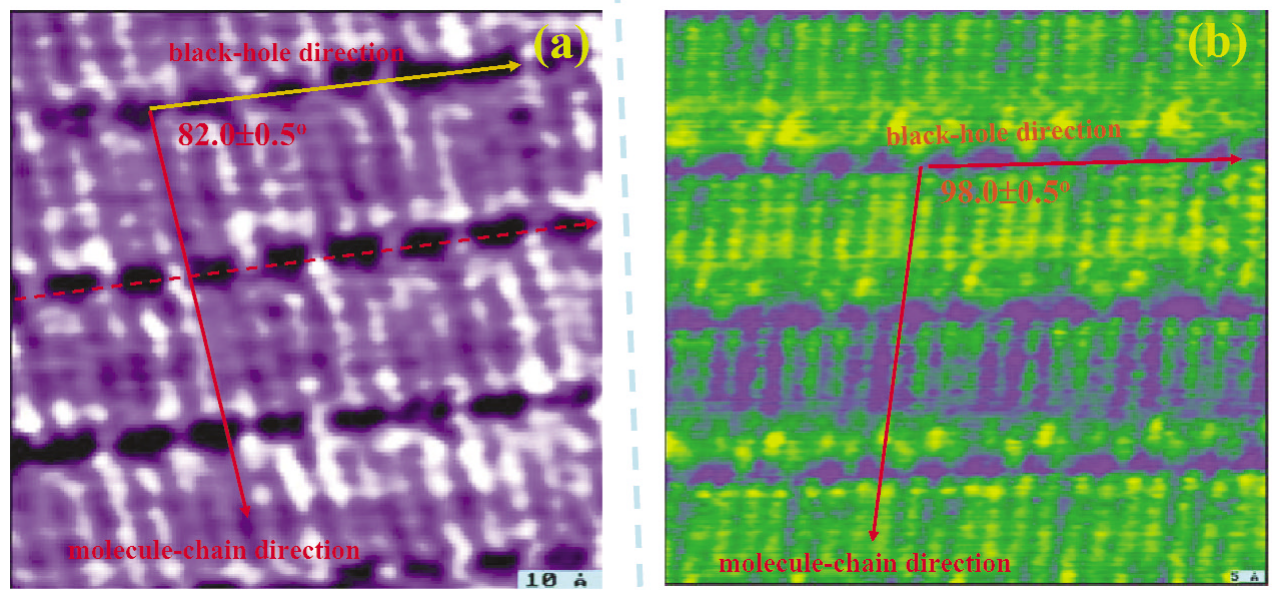

Fig. 7 Two kinds of domains ( $m$ and $m$ ') formed by self-assembly of achiral arachidic anhydride. The dimensions in images (a) and (b) are $72 \times 72 \AA$ and $93 \times 93 \AA$, respectively. The turquoise dashed line between images (a) and (b) is the mirror plane between the two enantiopure domains. Red dashed line shows the black-hole rows.

In addition, the hydrogen-bonding between adjacent molecular functional groups is another important factor in dominating molecular self-assembled structure. Recent studies show that the relative hydrogen-bond density between acid solvent molecules and $d i$-acid solute molecules determines whether a solute-solvent coadsorption occurs or not [13]. For example, octanoic acid (C8, solvent) has a larger hydrogen-bond density than HOOC- $\left(\mathrm{CH}_{2}\right)_{n}-\mathrm{COOH}(n=18)(\underline{\mathrm{C} 20}$, solute $)$; they coadsorb (Fig. 8b). However, this solvent has a lower hydrogen-bond density than HOOC- $\left(\mathrm{CH}_{2}\right)_{n}-\mathrm{COOH}(n=$ 12) ( $\mathrm{C} 14$, solute); they do not coadsorb (Fig. 8c). Systematic studies of solutes of different $d i$-acids and solvents of different acids demonstrated that acid solvent coadsorbs with $d i$-acid solute if the acid sol- 

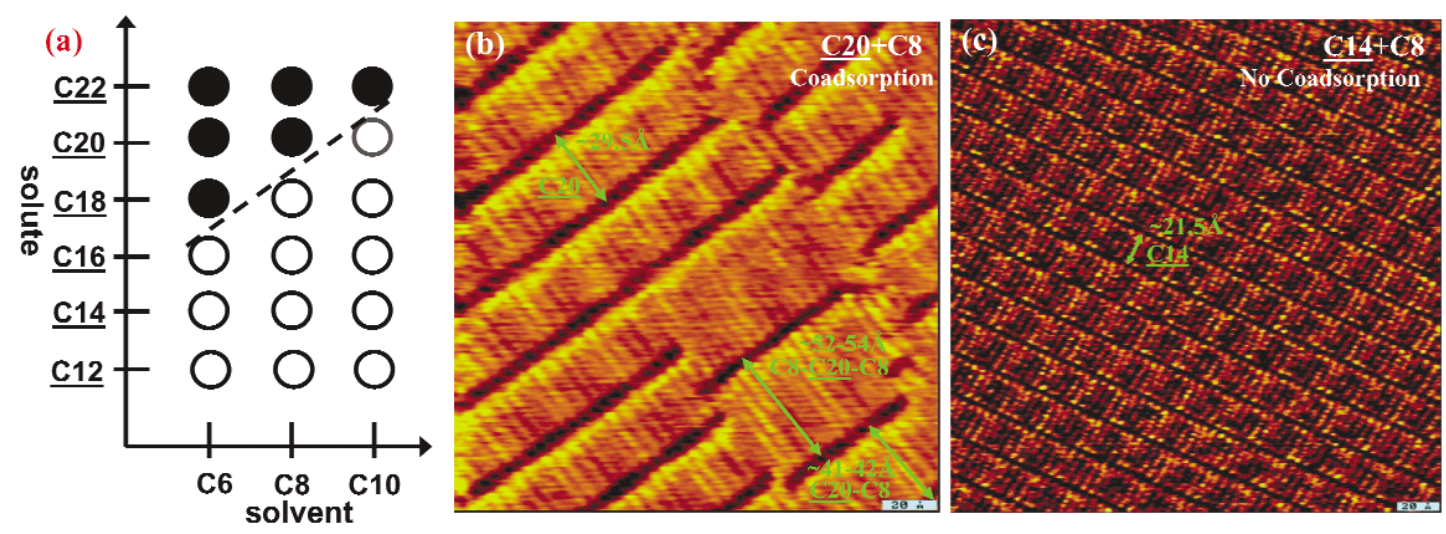

Fig. 8 (a) Coadsorption and relative hydrogen-bond density of solvent and solute molecules in organic selfassembled systems. Solid circles show coadsorption. Hollow circles show no coadsorption. (b) STM image showing coadsorption between acid solvent (C8) and $d i$-acid solute ( $\underline{\mathrm{C} 20})$ molecules. (c) STM image showing no coadsorption between acid solvent (C8) and $d i$-acid solute (14) molecules.

vent molecule has a larger hydrogen-bond density than the $d i$-acid solute molecule. Otherwise, they do not coadsorb. The driving force for the coadsorption is the maximization of hydrogen-bond density of the self-assembled monolayer as hydrogen-bonding is the main factor for the stability of the self-assembled monolayer. This finding can be used to direct synthesis of composite architectures from various solvents.

On the other hand, the intermolecular steric repulsion at the atomic level possibly results in distinctly different self-assembled structures and even induces different chirality for the self-assembled monolayers $[14,15]$. The simplest example is the odd-even effect on structure and chirality of carboxylic acid self-assembled on graphite (Fig. 9). $\mathrm{CH}_{3}\left(\mathrm{CH}_{2}\right)_{n-2} \mathrm{COOH}(n=$ odd) cannot form an enantiomer as there is a large repulsion between the hydrogen atom on the carboxylic acid group of one molecule and the hydrogen atom on the ending methyl group of the adjacent molecule, when two adjacent molecules use the same face to pack on the surface. Similarly, $\mathrm{CH}_{3}\left(\mathrm{CH}_{2}\right)_{n-2} \mathrm{COOH}(n=$ even) cannot form a racemic mixture as the hydrogen atom of the carboxylic acid group of one molecule is too close to one hydrogen atom of the ending methyl group of its adjacent molecule when two adjacent molecules use the opposite face to pack on the surface. Thus, $\mathrm{CH}_{3}\left(\mathrm{CH}_{2}\right)_{n-2} \mathrm{COOH}(n=$ odd) exhibits a different molecular packing pattern and chirality in contrast to $\mathrm{CH}_{3}\left(\mathrm{CH}_{2}\right)_{n-2} \mathrm{COOH}(n=$ even). Recent studies systematically revealed more odd-even effects on structures and properties of different organic architectures assembled on various solid surfaces [15]. These revealed odd-even effects are important for understanding structures and functions of the assembled organic architectures and devices. 

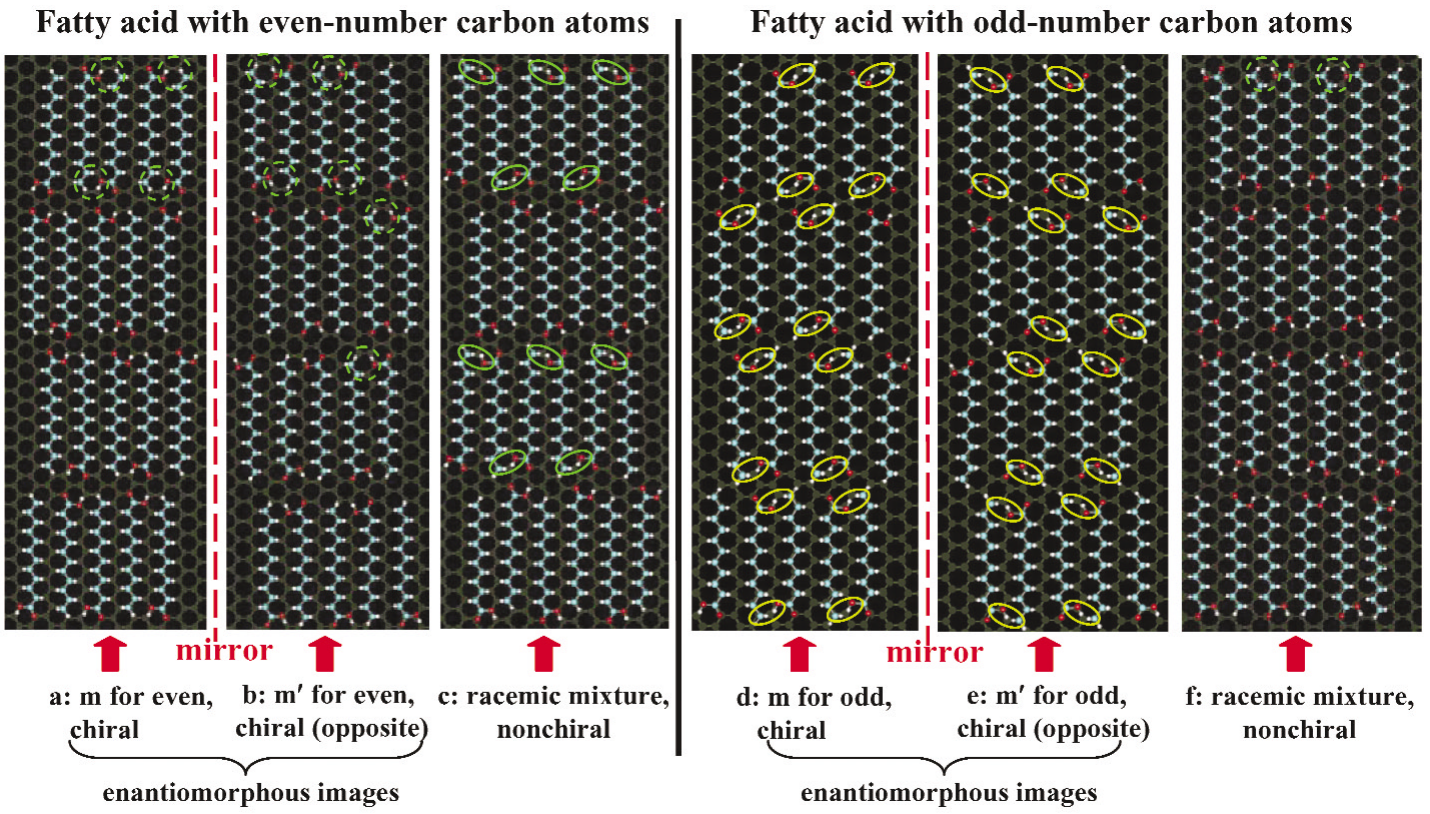

Fig. 9 Odd-even effect on molecular packing pattern and chirality for $n$-carboxylic acid. $\mathrm{CH}_{3}\left(\mathrm{CH}_{2}\right)_{10} \mathrm{COOH}$ and $\mathrm{CH}_{3}\left(\mathrm{CH}_{2}\right)_{9} \mathrm{COOH}$ represent even- and odd-acids, respectively. Even-acid forms enantiomorphous images with opposite chirality (Figs. 9a,b). Odd-acid forms a nonchiral racemic mixture (Fig. 9f).

\section{SYNTHESES OF NANOSTRUCTURED ORGANIC ARCHITECTURES ON SOLID SURFACES BY MOLECULAR SELF-ASSEMBLY VIA PRECISELY CONTROLLING WEAK NONCOVALENT INTERACTIONS}

Multicomponent organic architectures can offer multiple functionalities of organic materials and flexibility of fine-tuning chemical, physical, mechanical, and electronic properties of materials and devices. Based on the above understanding for the self-assembly of organic molecules at the atomic level, a new methodology to precisely and controllably grow ordered stoichiometric nanostructured multicomponent organic architectures was successfully developed. One example is the synthesis of a nanomesh via coself-assembly of multicomponent organic molecules by precisely controlling the nanoscale surface chemistry involved in this process [16].

Figure 10a is an STM image of 5-octadecyloxyisophthalic acid (5OIA) thin film. The superimposed molecular models in Fig. 10a and the molecular packing pattern in Fig. 10b clearly show the formation of an ordered and homogeneous single-component structure. For the organic architecture synthesized from 5OIA and octanoic acid, a distinctly different structure was obtained (Fig. 10c), in which 5OIA self-assembles with octanoic acid at the molecular level. The two molecules alternately pack in each lamella, forming a homogenous stoichiometric crystalline organic architecture. Nanoholes (marked with pink boxes in Figs. 10c and 10d) with a size of $13.5 \times 8.5 \times 1.8 \AA$ were formed due to the different chain-lengths of the two molecules, consistent with the line-profile analyses (Figs. 10e,f) for Sections 1 and 2 of Fig. 10c. The ordered homogeneous arrangement of nanoholes forms a homogeneous organic architecture of nanomesh. In addition, with this strategy a series of nanomeshes composed of nanoholes with different sizes were synthesized by using carboxylic acids with different lengths as the coadsorbed component. Figure $10 \mathrm{~g}$ is another example of a homogeneous multicomponent system of 5OIA and terephthalic acid.

This synthesis demonstrated a stoichiometric molecule-by-molecule co-self-assembly for fabricating nanostructured organic architectures on solid surfaces. This methodology also provides a prom- 

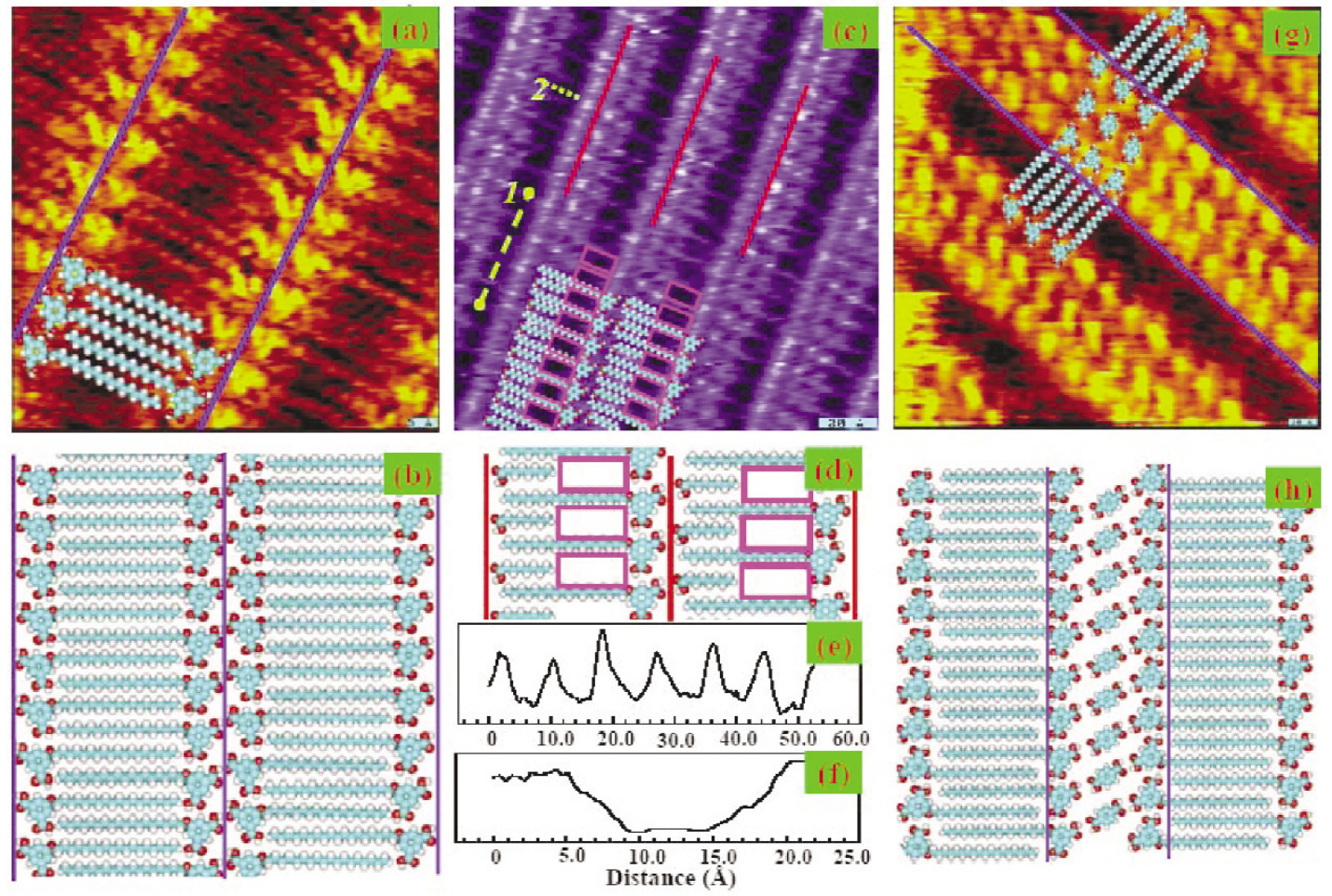

Fig. 10 (a) STM image of the self-assembled pure 5OIA. (b) Molecular packing pattern in this image. (c) STM image of nanomesh made of homogeneously arranged nanoholes. (d) Molecular packing pattern in this nanomesh. (e) and (f) Line-profile analyses for the size of the nanoholes. (g) STM image and (h) molecular packing pattern of co-self-assembled 5OIA and terephthalic acid.

ising approach to fabrication of conductive molecular wires/nanowires and nanocircuits on the surface of a solid substrate by finely controlling the weak intermolecular and molecule-substrate interactions in self-assembled systems.

\section{SURFACE CHEMISTRY AND REACTION MECHANISM IN DIRECTED-ASSEMBLY OF ORGANIC MOLECULES VIA STRONG CHEMICAL BONDS AND GROWTH OF ORGANIC ARCHITECTURES}

Compared to weak noncovalent interactions in molecular self-assembly, molecules are chemically bound to a well-defined solid surface with reactive sites in directed-assembly. The reactivity of molecular functional groups with the solid surfaces is extremely important in the directed-assembly. By assembling $b i$ - or multi-functional molecules via the formation of chemical bonds, solid surfaces can be modified and functionalized. The functionalized surface plays an important role in a wide spectrum of technological fields such as the development of new-generation microelectronics and biosensing techniques [17]. For example, the controllable growth of an organic monolayer and multilayer architecture on semiconductor surfaces is extremely important for designing biosensing devices utilizing the molecular recognition mechanism, because the assembled organic/biological molecules on the semiconductor surfaces can exhibit specific biocompatibility for certain biological molecules, proteins, or cells. A change of a specific physical property such as tunneling current can be used to monitor the specific biocompatibility. By identifying biocompatibility of protein or cell in this way, new diagnostic methods and sensing technologies could be developed. 
In the directed-assembly of organic molecules on semiconductor surfaces, two of the major semiconductor surfaces used are $\mathrm{Si}(111)-7 \times 7$ [18,19] and $\mathrm{Si}(100)$ [19]. Both of them are reagent-like reactive substrates. $\mathrm{Si}(100)$ undergoes a $2 \times 1$ reconstruction where two adjacent $\mathrm{Si}$ atoms pair up into dimers (Fig. 11a). This pairing results in the formation of a strong $\sigma$-bond and a weak $\pi$-bond between the two $\mathrm{Si}$ atoms within each dimer, making it structurally and electronically similar to the $\mathrm{C}=\mathrm{C}$ bond of an alkene. Organic molecules that contain one or more double and/or triple bonds can strongly bond on this surface through breaking the $\pi$-bonds of both the organic molecule and surface $\mathrm{Si}=\mathrm{Si}$ dimer to form two new $\sigma$-bonds at the organic/inorganic interface. This binding mode is valuable and unique because it does not involve the fragmentation of organic molecules and therefore provides better control of the molecular-level structures than dissociative reactions during functionalizing Si surfaces. In addition, the tilting of two silicon atoms of each dimer for the maximization of stability results in electron transfer from a tilted-down atom to a tilted-up one. This configuration makes the Si-dimer have both electron-rich and -deficient sites, offering sites for electron-deficient and -rich functional groups to form dative bonds, respectively (Fig. 11b). Another important semiconductor surface is $\mathrm{Si}(111)-7 \times 7$. Each $7 \times 7$ unit cell provides a number of reactive sites (Figs. 12a,b). Because of the large difference in electron density among surface atoms containing dangling bonds, an adatom coupled with one adjacent rest atom can act as a dipole-like reactive site (Fig. 12c). In addition, the electron-deficient adatom and electron-rich rest atom can be a binding site of electron-rich and -deficient functional groups to form dative bonds.

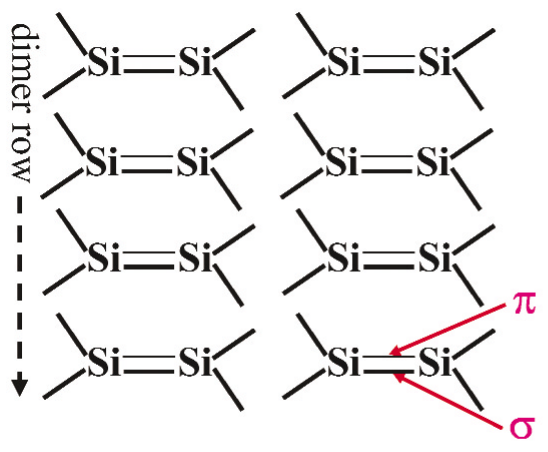

(a)

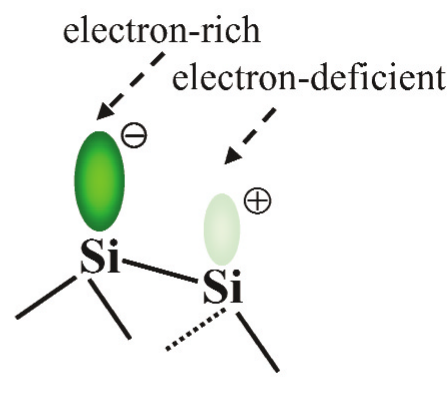

(b)

Fig. 11 (a) Reagent-like $\mathrm{Si}(100)$ made of homogeneously arranged $\mathrm{Si}=\mathrm{Si}$ dimers. (b) A tilted $\mathrm{Si}=\mathrm{Si}$ dimer made of one tilted-up electron-rich silicon atom and one tilted-down electron-deficient one.

The directed-assembly mechanisms of molecular functional groups on the two silicon surfaces including dissociation via $\mathrm{M}-\mathrm{H}(\mathrm{M}=\mathrm{O}, \mathrm{S}$, or $\mathrm{N})$ groups, formation of dative bond, step-wise [2+2]-like cycloaddition, and [4+2]-like cycloaddition, were revealed for different organic molecules [20-25]. Due to the large intermolecular separation determined by the homogeneously arranged reactive sites of solid surfaces and the strong chemical binding between molecule and reactive sites of these substrates, molecule-molecule interaction in one monolayer is quite weak. Thus, molecular packing in a directed-assembled monolayer is mainly determined by the distribution of reactive sites on the surface, which is significantly different from the intermolecular interactions-determined self-assembly through weak nonconvalent interactions. 


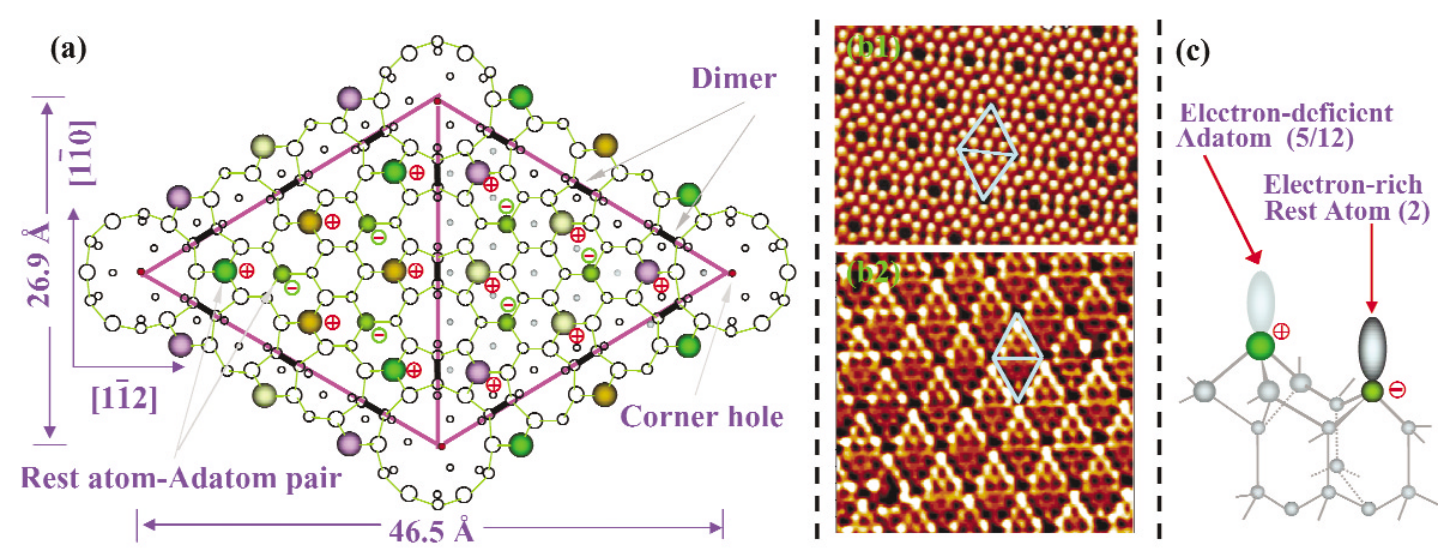

Fig. 12 (a) Structure of one unit-cell of $\mathrm{Si}(111)-7 \times 7$ surface. (b) High-resolution STM image of $\mathrm{Si}(111)-7 \times 7$ surface (b1 is image of unoccupied state; b2 is image of occupied state.). (c) One reactive site (adatom-rest pair) for directed-assembly of organic materials on this surface.

The competition and selectivity of different functional groups of the assembled multifunctional molecules were thoroughly investigated recently. For example, recent studies for the directed-assembly of complex aromatic molecules (Fig. 13) on $\mathrm{Si}(111)-7 \times 7$ discovered that the selection of reaction channel for these molecules is determined by the electronic contribution of heteroatoms for the formation of the aromatic conjugation of $(4 n+2) \pi$-electrons, the geometric arrangement and the electronegativity of the heteroatoms on the aromatic ring, and molecular polarity [26]. Based on the mechanistic understanding of the directed-assembly of multifunctional organic molecules on silicon surfaces, a novel layer-by-layer strategy could be designed and used for a controllable growth of organic multilayer architectures. This layer-by-layer assembly technique will possibly open up a door for controllably fabricating molecular multilayer architectures, which is crucial for a wide spectrum of technological applications.

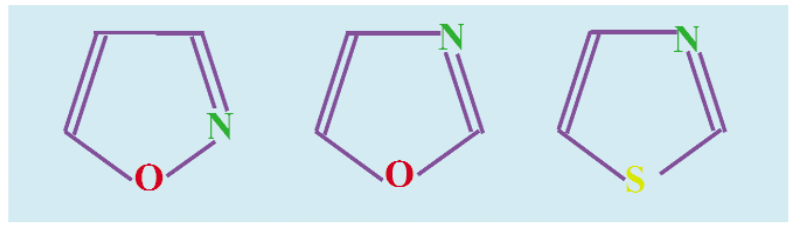

Fig. 13 Studied complex aromatic molecules.

\section{SUMMARY}

Nanoscale surface chemistry in the self- and directed-assembly of organic molecules on different solid surfaces was briefly reviewed for mechanistically understanding the structure and chirality created by these assembly techniques. On the basis of the atomic-level understanding of these assemblies, new synthetic methodologies for nanostructured organic architectures using these techniques were also discussed. For molecular self-assembly on solid surfaces through weak noncovalent interactions, molecule-substrate interactions could induce distortion of molecular configuration and even chirality. Molecule-molecule interactions, particularly interactions between functional groups, determine the molecular packing pattern by maximizing stability of the self-assembled monolayers, and could possibly be used to create macroscale 2D chiral materials. Directed assembly monolayers are formed through strong chemical binding between reactive sites of a substrate and molecular active functional groups. 
Molecular packing is dominated by competition and selectivity of different functional groups and the geometrical arrangement of reactive sites on the solid surface. Compared to the self-assembled monolayer formed through weak noncovalent interactions, intermolecular interactions in the directed-assembly monolayer do not play as important a role in determining molecular packing in a monolayer. Directed assembly of organic molecules on reactive $\mathrm{Si}(100)$ and $\mathrm{Si}(111)-7 \times 7$ was taken as example for mechanistically understanding directed-assembly at the atomic level. Reaction mechanisms of the directed-assembly on silicon surfaces were discussed. The understanding of molecular self- and directed-assembly at the atomic scale will definitely aid the development of new 2D and 3D organic architectures for significant applications in a wide spectrum of technological fields.

\section{ACKNOWLEDGMENT}

The author expresses his deepest appreciation to his advisor, Prof. Steven L. Bernasek, for his excellent education, tremendous support, and constant encouragement in his graduate career at Princeton University.

\section{REFERENCES AND NOTES}

1. F. Tao. Ph.D. dissertation, Princeton University (2006).

2. F. Tao, Y. Cai, S. L. Bernasek. Langmuir 21, 1269 (2005).

3. F. Tao, S. L. Bernasek. Langmuir 23, 3513 (2007).

4. G. C. McGonigal, R. H. Bernhadt, D. J. Thomson. Appl. Phys. Lett. 57, 28 (1990)

5. G. Watel, F. Thibaudau, J. Cousty. Surf. Sci. 281, L297 (1993).

6. G. Quinkert, E. Egert, C. Griesinger. Aspects of Organic Chemistry and Structure, VCH, New York (1996).

7. The energies of the $a p$ - and $s p$-conformations, and the structure upon linear distortion were calculated with Gaussian 98 at the theory level of B3LYP with a basis set of 6-31G(d).

8. W. Liang, M. H. Whangbo, A. Wawkusschewski, H. J. Kantow, S. N. Magnov. Adv. Mater. 5, 817 (1993).

9. C. L. Claypool, F. Faglioni, W. A. Goddard, W. B. Gray, N. S. Lewis, R. A. Marcus. J. Phys. Chem. B 101, 5978 (1997).

10. Y. Cai, S. L. Bernasek. J. Am. Chem. Soc. 126, 14234 (2004).

11. B. Venkataraman, J. J. Breen, G. W. Flynn. J. Phys. Chem. 99, 6608 (1995).

12. F. Tao, S. L. Bernasek. J. Phys. Chem. B 109, 6233 (2005).

13. F. Tao, J. Goswami, S. L. Bernasek. J. Phys. Chem. B 110, 19562 (2006).

14. F. Tao, J. Goswami, S. L. Bernasek. J. Phys. Chem. B 110, 4199 (2006).

15. F. Tao, S. L. Bernasek. Chem. Rev. 107, 1408 (2007).

16. F. Tao, S. L. Bernasek. J. Am. Chem. Soc. 127, 12750 (2005).

17. J. T. Yates. Science 275, 339 (1998).

18. H. N. Waltenburg, J. T. Yates. Chem. Rev. 95, 1589 (1995).

19. K. Takayanagi, T. Tanishiro, S. Takahashi, M. Takahashi. J. Vac. Sci. Technol. A 3, 1502 (1985).

20. R. J. Hamers, S. K. Coulter, M. D. Ellison, J. S. Hovis, D. F. Padowitz, M. P. Schwartz, C. M. Greenlief, J. N. Russell. Acc. Chem. Res. 33, 617 (2000).

21. S. F. Bent. J. Phys. Chem. B 106, 2830 (2002).

22. S. F. Bent. Surf. Sci. 500, 879 (2002).

23. M. A. Filler, S. F. Bent. Prog. Surf. Sci. 73, 1 (2003).

24. R. A. Wolkow. Annu. Rev. Phys. Chem. 50, 413 (1999).

25. F. Tao, G. Q. Xu. Acc. Chem. Res. 37, 882 (2004).

26. F. Tao, S. L. Bernasek. J. Am. Chem. Soc. 129, 4815 (2007). 